\title{
Lysosomal Acidification Mechanisms
}

for Annual Reviews in Physiology

Joseph A. Mindell

Membrane Transport Biophysics Section National Institute of Neurological Disorders and Stroke/NIH 35 Convent Drive, Bldg 35, MSC 3701

Bethesda, MD 20892

mindellj@ninds.nih.gov 


\begin{abstract}
Lysosomes are the terminal organelles on the endocytic pathway, digesting macromolecules and making their components available to the cell as nutrients. Hydrotlytic enzymes specific to a wide range of targets reside within the lysosome; these enzymes are activated by the highly acidic $\mathrm{pH}$ (between 4.5 and 5.0) in the organelles' interior. Lysosomes generate and maintain their pH gradients using the activity of a proton-pumping V-type ATPase, which uses metabolic energy in the form of ATP to pump protons into the lysosome lumen. Since this activity separates electric charge and generates a transmembrane voltage, another ion must move to dissipate this voltage for net pumping to occur. This so-called counterion may be either a cation (moving out of the lysosome) or an anion (moving in). Recent data supports the involvement of $\mathrm{ClC}-7, \mathrm{a} \mathrm{Cl}^{-} / \mathrm{H}^{+}$antiporter in this process, though many open questions remain as to this transporter's involvement. Though functional results also point to a cation transporter, its molecular identity remains uncertain. Both the V-ATPase and the counterion transporter are likely to be important players in the mechanisms determining the steady state $\mathrm{pH}$ of the lysosome interior. Exciting new results suggest that lysosomal $\mathrm{pH}$ may be dynamically regulated in some cell types.
\end{abstract}




\section{Contents}

Lysosomal Acidification Mechanisms.....................................................................................................

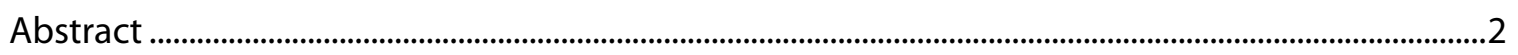

Lysosomal Acidification Mechanisms................................................................................................4

The V-type Proton ATPase ...................................................................................................................

Structure and mechanism ....................................................................................................................

The V-ATPase is electrogenic ......................................................................................................

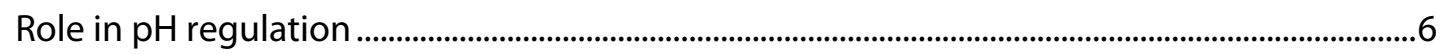

Counterion movement and lysosomal acidification ...............................................................................

The counterion pathway in isolated lysosomes......................................................................................

The counterion pathway in lysosomes in "intact" cells .......................................................................

The counterion pathway in the molecular era .............................................................................................

The Cystic Fibrosis Transmembrane Regulator ...............................................................................9

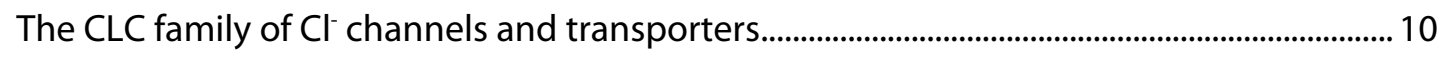

Could ClC-7 be a lysosomal counterion pathway?....................................................................... 10

Role of proton-chloride coupling in CIC-7 function ...................................................................... 11

New possibilities: active regulation of lysosomal $\mathrm{pH}$...................................................................... 12

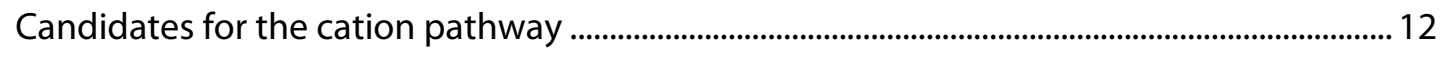

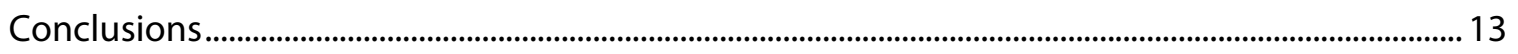

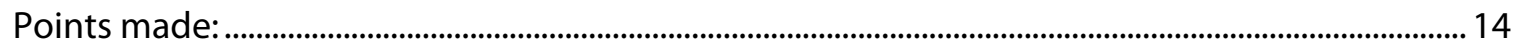

Future directions: ..................................................................................................................................... 14

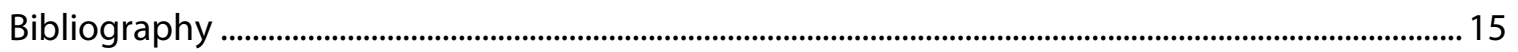




\section{Lysosomal Acidification Mechanisms}

Mammalian cells use $\mathrm{H}^{+}$for a broad range of physiological functions, not surprising given the ubiquity and reactivity of the bare proton (or hydronium ion). Protons lie at the center of bioenergetics, since mitochondria use a gradient of these ions across their inner membranes as a key intermediate in oxidative phosphorylation. Perhaps taking a cue from these cellular batteries, a wide range of other membrane-bound intracellular organelles also generate transmembrane proton gradients, including the Golgi apparatus, secretory vesicles, endosomes and lysosomes (Figure 1). Key cellular processes depend on the lumenal $\mathrm{pH}$ inside given organelles, including posttranslational modification in the secretory pathway, ligand targeting in the endosomal pathway, and macromolecule degradation in the lysosome. Each organelle maintains a characteristic internal pH, which is essential for facilitating its function. Indeed, disorders that affect organellar acidification can lead to a range of diseases, many of which are severe or life-threatening. Perhaps the most extreme example of organellar acidification in mammalian cells is the lysosome. This digestive organelle depends on maintaining a highly acidic $\mathrm{pH}$ (below $\mathrm{pH}$ 5.0) in its lumen to successfully perform its digestive function and to drive efflux of digested materials.

Evidence for an acidic lysosomal lumen initially came from the convergence of multiple approaches. Actually, the earliest evidence for acidification of intracellular compartments predates the discovery of the lysosome. In 1893, Metchnikoff found that paramecia could ingest particles of $\mathrm{pH}$ sensitive litmus paper, and that upon internalization the paper changed color consistent with entering an acidic compartment(1). In the modern era, Early indications of of acidic lysosomes in the modern era came from observations by De Duve, who noted that the hydrolytic enzymes contained in the lysosome share acidic $\mathrm{pH}$ optima(2). Studies on the distribution of radioactively or fluorescently labeled weak bases (like acridine orange) on isolated lysosomes provided direct evidence that the organelles could maintain an internal $\mathrm{pH}$ more acidic than the bathing medium.

It is now well-established that this $\mathrm{pH}$ gradient is generated by the action of a V-type ATPase, a proton-pumping membrane protein that uses the free energy of ATP hydrolysis to drive protons against their electrochemical gradient into the lysosome lumen(3). However, with each proton pumped the ATPase also generates a voltage difference across the lysosome membrane, which inhibits further pumping. Thus, for the ATPase to effectively acidify the lysosome interior, proton movement must be accompanied by the movement of a counterion to dissipate the transmembrane voltage generated by the ATPase. In theory, this counterion movement could be generated either by entry of a cytoplasmic anion into the lysosome interior or by exit of a cation from the lumen to the cytoplasm (Figure 2). The identity of this counterion remains controversial. Yet the process of counterion movement may be an important element of the acidification process; though many organelles use the same v-type ATPase to acidify their interiors, each is able to maintain a stable, characteristic internal $\mathrm{pH}$; varying counterion mechanisms may account for this. The mechanisms of this $\mathrm{pH}$ regulation are poorly understood, especially at a quantitative level. Since the movement of counterions is essential for acidification, the molecules involved present potential elements of such organellar pH-regulatory mechanisms. The lysosome is potentially an ideal organelle to probe these mechanisms, since it is relatively easily studied both in living cells and in isolation. Here, I will review the known and hypothesized elements of the lysosomal acidification mechanism and consider the experimental results providing insight into to the counterion movement. I will also highlight exciting new results that could pertain to ultimately understanding the regulation and maintenance of lysosomal $\mathrm{pH}$. 


\section{The V-type Proton ATPase}

The primary driver of acidification throughout the endocytic pathway, including in the lysosome, is the V-type proton ATPase, harvesting free energy from ATP hydrolysis to drive protons uphill into the lysosome. The V-type ATPase is structurally similar to the $F_{0} F_{1}$ ATPases involved in mitochondrial oxidative phosphorylation. However, where the $F_{0} F_{1}$ ATPases can either synthesize or hydrolyze ATP, the v-type ATPases appear to be optimized for proton pumping, since they apparently only work in the hydrolytic direction in vivo.

\section{Structure and mechanism}

The V-ATPases are multisubunit complexes composed of a soluble, $V_{1}$ subcomplex (analogous to the $F_{1}$ portion of the $F$-ATPase) and a membrane-embedded $V_{0}$ subcomplex (analogous to $\left.F_{0}\right)(4)$. Each subcomplex is composed of multiple protein subunits (Figure 3 ). The soluble $V_{1}$ domain includes at least 8 subunits $(A-H)$ and includes the loci catalyzing ATP hydrolysis. The membrane-embedded $V_{0}$ subcomplex includes subunits $a, c, c^{\prime}, c^{\prime \prime}, d$, and e, and is required for protein translocation across the membrane. In the intact complex, the $\mathrm{V}_{1}$ and $\mathrm{V}_{0}$ portions are connected by a central "stalk" similar to those seen in the $F_{0} F_{1}$ ATPase (including $D$, and $F$ subunits) as well as several peripheral stalks ( 3 in most eukaryotic $V$-ATPases, formed by $E$ and $G$ subunits).

Also like the $F_{0} F_{1}$ ATPases, the V-ATPase is a rotary proton-transport motor. Hydrolysis of ATP at the interfaces between $A$ and $B$ subunits in the catalytic domain, drives the rotation of the central stalk, a 'driveshaft' $(5,6)$. The stalk is coupled to the proton translocating ring of $c$ subunits, located within the membrane, which, in turn, mediates proton translocation. Protons are thought to ride around the c-based rotor on the acidic sidechain of a strictly conserved glutamate residue located midway along a membrane spanning helix on each c subunit. The protons access this group through a pair of "hemi-channels," each providing an aqueous pathway halfway through the membrane through different regions of the a subunit(4). A conserved positive arginine resides between the hemichannels, forcing the proton to take the long way around the rotor and presumably helping it dissociate through the luminal hemichannel. This mechanism can explain proton pumping by the ATPase: the rotor is driven uniquely in one direction by the irreversible hydrolysis of ATP, protons only access the rotor from the cytoplasmic-facing hemichannel and only leave it from the lumen-facing hemichannel, resulting in unidirectional proton flux.

The ability of a v-type ATPase to generate a pH gradient depends critically on the ratio of ATP hydrolysis to proton transport. In this respect, the V-ATPases seem to be optimized for proton pumping compared to the $\mathrm{F}_{0} \mathrm{~F}_{1}$ ATPases which can be driven in either direction (ATP synthesis or hydrolysis) in vivo. With a hexameric A/B complex with 3 ATP binding sites, and the likely 6membered ring formed by the 3 varieties of proton-translocating $c$ subunits (including $c, c^{\prime}$ and $\left.c^{\prime \prime}\right)(7)$, a stoichiometry of 2 protons translocated per ATP hydrolyzed is expected. Such stoichiometries have indeed been measured using both kinetic and thermodynamic methods $(8,9)$. Based on the free energy of ATP hydrolysis, an ideal proton ATPase that pumps 2 protons/ATP could generate the proton gradient of more than four $\mathrm{pH}$ units(10). Indeed, very large $\mathrm{pH}$ gradients can be generated by the ATPase in the vacuoles of citrus fruits, near the ideal gradient(11), though other transporters may also be involved in this system.

\section{The V-ATPase is electrogenic}

On theoretical grounds, a transporter that moves a single ion unidirectionally across the membrane should build up a gradient of across that membrane, i.e. a voltage difference. This voltage should be of a sign such that it inhibits further transport-ultimately the voltage should build to the point that net transport is fully inhibited and no further flux occurs (absent dissipation of that charge 
by some counterion movement, see below). As a unidirectional proton transporter, thus, the V-ATPase is predicted to act in this way, to be electrogenic. This prediction was first tested using voltagesensitive probes, like diS- $\mathrm{C}_{3}-(5)$, a member of the cyanine family. These hydrophobic cationic fluorescent dyes accumulate in compartments with negative transmembrane voltages, quenching their fluorescence. Using these dyes, Harikumar and Reeves, and Okhuma and colleagues demonstrated that where isolated lysosomes initially showed negative membrane potentials, these voltages became more positive upon addition of ATP to activate the V-ATPase(12, 13). An active electrogenic transporter is also expected to generate electrical currents across its membrane, a prediction verified for both plant(14) and yeast transporters(15) using patch clamp techniques. These methods clearly establish the electrogenicity of the V-ATPase. As noted above, for an electrogenic transporter to effectively move its substrate the voltage it generates must be dissipated by another mechanism: possible mechanisms for this dissipation in lysosomes will be discussed below.

\section{Role in $\mathrm{pH}$ regulation}

The V-type ATPase generates different $\mathrm{pH}$ gradients in different organelles and on the plasma membrane. Though the mechanisms that set these $\mathrm{pHs}$ remain unknown, a reasonable hypothesis is that at least part of these differences may be accounted for by regulation of the ATPase itself. Several forms of regulation have been observed for the V-ATPase protein complex, though none of them has been shown to directly influence organellar acidification.

The most dramatic form of regulation yet observed for a V-type ATPase is the reversible dissociation of the enzyme complex observed in both yeast and the tobacco hornworm, Manduca Sexta. In both cases, nutrient restriction (glucose deprivation in yeast, molt or starvation in Manduca) led to the dissociation of the V-ATPase into membrane-embedded V0 and soluble V1 $(16,17)$. Notably, this process, which turns off both ATPase activity and proton translocation is completely reversible with the restoration of nutrients in yeast(17). Furthermore, the process is not dependent on translation of new protein (17). The ATPase dissociation is modulated by interactions with other proteins and protein complexes(18-20), including the glycolytic enzyme aldolase(21), as well as with microtubules(22).

In addition to these metabolic regulatory mechanisms, targeting of the ATPase can be regulated by varying its subunit composition. This process has been characterized in detail in yeast, where all of the ATPase subunits are coded by single genes except for the a subunit, which has two coding genes. These two genes, VPH1 and STV1, are 54\% identical but have very different effects on the complex; where Vph1p targets the ATPase to vacuoles, Stv1p targets the complex instead to the Golgi complex $(23,24)$. When expressed in a strain with both STV1 and VHP1 genes disrupted, overexpression of the Golgi-targeted Stv1p results in some targeting of the V-ATPase complex to the vacuole membrane, allowing direct comparison of the functional properties of the two ATPase isoforms(24). Forgac's group took advantage of this to compare the functional properties of the two isoforms in an otherwise very similar environment(25). They found that the two isoforms had similar kinetic properties, both showing $\mathrm{K}_{\mathrm{M}}$ for ATP of $\sim 250 \mu \mathrm{M}$. An $\sim 8$-fold reduction in $\mathrm{V}_{\max }$ was attributed to fewer assembled ATPase complexes. The property most pertinent here is the coupling of ATP to proton transport, with Stv1 p-containing complexes showing a 4-5-fold lower coupling of ATP hydrolysis to proton transport compared with Vph1p-containing complexes. Thus, the ATPase can 'slip'; it can hydrolyze ATP without pumping protons. Such a mechanism seems waseful of ATP, but the observation is consistent with the lower $\mathrm{pH}$ observed in the vacuole compared with that in the Golgi.

Like the yeast protein, mammalian V-ATPase subunits are encoded by multiple genes and the complexes are found with a range of isoforms (reviewed in (26)). Generally, a single isoform of each subunit is widely expressed with alternate subunits expressed in limited ranges of tissues. For 
example, the $\mathrm{d} 1$ subunit is ubiquitously expressed, whereas the $\mathrm{d} 2$ is expressed at high levels only in osteoclasts, kidney and lung(27). Indeed, the phenotype of mice with knockouts of the $\mathrm{d} 2$ subunit were limited to bone, where the animals had osteopetrosis, an increased bone density(28). Other subunit isoforms are highly expressed in kidney tubules and in inner ear(29), both tissues with highly specialized transport needs. Notably, though, for each of these examples the alternate subunit is associated with an unusual targeting of the V-ATPase to the plasma membrane, rather than to an alternate organelle (as is observed in yeast). To this author's knowledge there are no known cases of mammalian V-ATPase isoforms associated with targeting to alternate organelles (reviewed in (26)). Thus, the question remains open of whether the V-ATPase, the essential driver of organellar acidification, is directly involved in the determination and regulation of organellar $\mathrm{pH}$ in general and of lysosomal $\mathrm{pH}$ in particular. To understand the mechanism of organellar $\mathrm{pH}$ regulation, we must consider other possible mechanisms.

\section{Counterion movement and lysosomal acidification}

As noted above, for a V-ATPase to effectively acidify an organelle, its action must be supplemented with a mechanism to dissipate the luminal-positive transmembrane voltage it generates. The general mechanism is described by the term "counterion pathway." Two general mechanisms could provide this dissipation: either a cation permeability could carry cations out of the organelle, with one cation removed for each proton translocated; alternatively, an anion permeability could move an anion into the lumen for each proton. A combination of these mechanisms could also be effective. Experimental analysis of lysosomal counterion pathways has a long and varied history. Many of the pioneering studies of lysosomal pH examined ion effects on acidification in isolated lysosomes. These results often pertain to understanding the counterion pathway, though they yield limited insight into the molecular basis of this path. More recently, a number of channels and transporters have been proposed to play roles in the counterion mechanism. However, the identity of the counterion remains controversial, as I will discuss below. Nevertheless, if counterion movement is a rate-limiting step in the acidification process then the counterion pathway could be extremely important in the regulation of lysosomal $\mathrm{pH}$.

\section{The counterion pathway in isolated lysosomes}

Many of the pioneering papers that explored the lysosomal acidification process presented data relevant to understanding the role of counterions in this mechanism. In a 1979 study of acidification in isolated lysosomes using the fluorescent weak base Acridine Orange as an indicator, Dell'antone found that substituting external $\mathrm{Cl}^{-}$with $\mathrm{SO}_{4}{ }^{2-}$ completely inhibited dye accumulation (and hence, acidification), an effect reversed by adding $\mathrm{Cl}^{-}$back to the bathing medium(30). Later, (in the study that firmly established that lysosomal acidification is driven by an ATPase) Okhuma et al. prepared lysosomes containing a fluorescent dextran derivatives by injecting the labeled-dextrans into rats and subsequently isolating lysosomes from those animals(3). After a short time, the dextran accumulates exclusively in lysosomes and the fluorescein moiety provides a $\mathrm{pH}$-dependent fluorescent signal. In these experiments, removal of external $\mathrm{Cl}^{-}$dramatically slowed acidification, whereas external cation replacement had little effect, supporting the involvement of anions as counterions. Of course, a cation-conducting counterion path would be moving cations out of the lysosomal lumen, but even in this case, changing the $\mathrm{K}^{+}$gradient might be expected to affect the rate of cation exit and therefore the acidification rate.

Further information on the role of cations came from experiments adding the $\mathrm{K}^{+}$ionophore valinomycin and the $\mathrm{H}^{+}$ionophore FCCP in different orders. Each of these agents makes the lysosome membrane highly and specifically permeable to its ion of choice. In experiments adding these ionophores, Ohkuma et al.(3) found that adding either one alone had minimal effects on lysosomal pH. 
In contrast, adding both agents rapidly dissipated the $\mathrm{pH}$ gradient. If there was a substiantial $\mathrm{K}^{+}$ permeability in the lysosome membrane before valinomycin addition, then FCCP alone should have dissipated the $\mathrm{pH}$ gradient. Together, these experiments suggest that anion movements are the primary path for counterions in this preparation.

Similarly, experiments measuring the lysosomal voltage point to significant $\mathrm{Cl}^{-}$permeability and limited $\mathrm{K}^{+}$permeability. Using the voltage sensitive dye, DiS- $\mathrm{C}_{3}-(5)$, Harikumar and Reeves found small but measurable effects of $\mathrm{K}^{+}$addition on dissipating the lysosomal membrane potential, where $\mathrm{Cl}^{-}$and other anions were more effective(13). Though similar experiments by Ohkuma et al. revealed a higher $\mathrm{K}^{+}$permeability, they were performed with tritosomes, lysosomes with altered buoyant density resulting from injection of triton WR-1339 into rats. The authors point out that this manipulation changes the $\mathrm{K}^{+}$permeability; the ion is much less permeant in untreated lysosomes(12). Finally, using the same dye, Cupolletti and coworkers found that ATP addition induced a large positive shift in membrane potential which was reversed in a concentration-dependent manner by addition of $\mathrm{Cl}^{-}$but not by $\mathrm{SO}_{4}^{-2}(31)$. A thorough study of endosome and lysosome acidification by Van Dyke supports the previous general conclusions regarding the counterion pathway(32), with acidification possible but slowed in media with either $\mathrm{K}^{+}$or $\mathrm{Cl}^{-}$replaced with an impermeant ion and a generally low permeability of the lysosomal membrane to other physiological ions. Together, these experiments support the conclusion that both $\mathrm{Cl}$ - and $\mathrm{K}^{+}$facilitate lysosomal acidification, with $\mathrm{Cl}^{-}$perhaps the primary ion contributing in some circumstances and both ions contributing in others.

Remarkably, despite many demonstrations of important roles for $\mathrm{Cl}^{-}$in lysosomal acidification, none of these studies directly demonstrated $\mathrm{Cl}^{-}$flux in the organelle. Such flux was recently established (33) in isolated rat liver lysosomes and was shown, surprisingly, to result from not the action of a $\mathrm{Cl}^{-}$, as had been assumed, but by a transporter exchanging two $\mathrm{Cl}^{-}$ions moving in one direction for a single proton moving the opposite direction. These experiments also revealed that this $\mathrm{Cl}^{-} / \mathrm{H}^{+}$antiporter is the primary pathway for $\mathrm{Cl}^{-}$movement across the lysosomal membrane and facilitated identification of the molecular basis for this activity, as I will discuss below.

In summary, multiple studies using isolated lysosomes to examine functional properties of the lysosomal acidification process agree that the primary permeability of native lysosomal membranes is to monovalent anions and cations, with both ions potientially serving as counterions for the V-ATPase. The relative contributions of these ions remain to be clearly established, but promising new methods may provide means to determine these.

\section{The counterion pathway in lysosomes in "intact" cells}

Most efforts to probe lysosomal counterion conductances have used isolated lysosomes. These preparations have great advantages insofar as the primary membrane in sample is lysosomal and that the ionic compositions at least outside, and sometimes on both sides, of the membrane can be well controlled. However, the ideal situation to study acidification is in lysosomes within living cells. Studies of lysosomal ion dynamics in vivo have been limited by the difficulty of adjusting cytoplasmic conditions across an intact plasma membrane. Recent work from Grinstein's lab(34) sought to improve the study of lysosomes in intact cells (RAW macrophages) by using a creative approach to breach the plasma membrane. These cells natively express $\mathrm{P}_{2} \mathrm{X}_{7}$ receptor, an ATP-activated ion channel(35). Under normal activating conditions, $\mathrm{P}_{2} \mathrm{X}_{7}$ receptors are ligand-activated, cation-selective ion channels. However, upon prolonged stimulation with ATP, these channels open a larger pore, possibly formed by a pannexin protein, which is permeable to molecules up to $\sim 900$ Daltons(36). By activating $P 2 X_{7}$ in RAW cells and bathing in varying solutions the investigators could dialyze the cytoplasm and significantly alter the cells' ion compositions.

Using this method, Grinstein's group attempted to test the importance of $\mathrm{Cl}^{-}$for lysosomal acidification by dialyzing into their cells a $\mathrm{Cl}^{-}$-free medium. Under these conditions, lysosomes whose 
$\mathrm{pH}$ gradients had been dissipated with the proton ionophore FCCP could reacidify equally well with our with $\mathrm{Cl}^{-}$in the bathing medium. Based on this observation, the investigators concluded that cytoplasmic $\mathrm{Cl}^{-}$is not required for lysosomal acidification. However, quantitative chemical analysis revealed nearly $10 \mathrm{mM}$ residual $\mathrm{Cl}^{-}$remaining the dialyzed cytoplasm of the " $\mathrm{Cl}^{-}$free" cells. Based on the $\mathrm{Cl}^{-}$-dependence of acidification(32) this is likely to be a sufficient concentration to serve as counterion.

Further experiments using the $\mathrm{P} 2 \mathrm{X}_{7}$ system explored the role of cations in lysosomal acidification. However, since the relevant ions in this case would reside in the lysosome lumen, further manipulations were required to manipulate their concentrations. To change luminal cation concentrations, the investigators permeablized the plasma membrane as described above, bathed the cells in solutions containing altered cation concentrations, resealed the PM by deactivating the P2X recptors, then added a dipeptide Gly-Phe- $\beta$-naphthylamide (GPN) to the cells. This membrane permeant peptide diffuses into the cells and into lysosomes, where it is cleaved by the peptidase Cathepsin C (only found in lysosomes)(37). The product are thought to accumulate in lysosomes and, since they are osmotically active, cause the organelles to swell and partially rupture, thereby allowing the cytoplasmic solution to equilibrate with the lysosomal interior(37, 38). Remarkably, removal of the peptide seems to restore lysosomal integrity and to allow the organelles to reacidifiy(34). Using these methods, Grinstein and colleagues determined that removing permeant cations (primarily $\mathrm{K}^{+}$) from the lysosome raised its luminal pH significantly, supporting earlier indications of a cation conductance contributing to lysosomal acidification. Though the methods used in this paper require futher validation, and may be limited in applicability to cell types expressing the appropriate proteins, the general approach pioneered by Steinberg et al. is very promising, offering the possibility of studying detailed lysosomal transport mechanisms in conditions approaching their native state.

\section{The counterion pathway in the molecular era}

The results discussed above reveal a great deal about the functional aspects of the counterion conductance, but they do not directly address the molecular identity of the transporters and channels that might be involved. Over the past two decades, a variety of molecules, including both chloride channels and transporters and cation channels, have been proposed to play this role, with varying degrees of experimental support. ,

\section{The Cystic Fibrosis Transmembrane Regulator}

The Cystic Fibrosis Transmembrane Regulator (CFTR) is the protein mutated in patients with the disease cysteic fibrosis. This protein is a member of the very broad ATP-binding cassette (ABC) transporter family, members of which use ATP hydrolysis to drive a huge array of substrates uphill against their concentration gradients(39). CFTR, however, is unique among $A B C$ transporters, as an ATP and phoshphorylation-activated $\mathrm{Cl}^{-}$channel, facilitating downhill anion flux(40). As the first chloride channel to be identified at the molecular level, CFTR proved to be a tempting candidate for an organellar anion shunt. However, it has had a checkered history in that role (nicely reviewed in (41)). Over the years several groups have reported observed changes in organellar pH in cells from CF patients (42) or in phagosomes from $\mathrm{Cftr}^{-/}$mice $(43,44)$. However, further work has questioned these results with several careful studies revealing no changes due to CFTR mutation in endosomes and Golgi(45-47). The methodology used in the study of phagosomal CFTR was also questioned and further examination revealed no $\mathrm{pH}$ change in phagosomes in several cell lines with pharmacologic inhibition of CFTR or knockout of the gene $(48,49)$. Furthermore, the limited tissue distribution of the CFTR protein could at best account for acidification in a very limited subset of lysosomes. Given the 
overall picture, CFTR is unlikely to be an important player in organellar acidification, particularly in the lysosome.

\section{The CLC family of $\mathrm{Cl}^{-}$channels and transporters}

Another candidate for the anion conductance in intacellular organelles, including the lysosome is the $\mathrm{CLC}$ family of $\mathrm{Cl}^{-}$channels and transporters. The first known member of this family was originally identified as a voltage-dependent chloride channel in the electric organ of the Torpedo ray by Chris Miller(50) and cloned by Thomas Jentsch (51). Since that time, the CLC's have grown to be the largest known family of chloride transporters, with nine known mammalian isoforms as well as homologs in yeast, plants, invertebrates, and a wide range of bacteria and archaea (52). CLC proteins have been implicated in a range of human diseases, from myotonia to disorders of renal transport to osteopetrosis(53). X-ray structures of bacterial (54) and eukaryotic (55) CLCs reveal the proteins to be homodimers, consistent with functional (56) and biochemical (57) data. Recently, Accardi and Miller found that a bacterial CLC homolog is not a channel at all, but rather a $\mathrm{Cl}^{-} / \mathrm{H}^{+}$antiporter, coupling the downhill movement of two $\mathrm{Cl}^{-}$ions to the uphill movement of a single proton (or vice versa) $(58,59)$. Indeed, some of the mammalian CLC isoforms are also antiporters $(60,61)$. Notably, these proteins, $\mathrm{ClC}-4$ and $\mathrm{CIC}-5$ are localized to endosomes and have been proposed to serve as counterion pathways in that organelle(62-64).

\section{Could CIC-7 be a lysosomal counterion pathway?}

Members of the $\mathrm{CLC}$ family of $\mathrm{Cl}$ - channels and transporters were first proposed to have a role in lysosomal acidification based on the results of a mouse knockout study of $\mathrm{ClC}-7$ from the Jentsch lab(65). ClC-7 (and the closely-related CIC-6) was cloned in 1995 and were shown to be broadly expressed, based on Northern Blots(66). However, neither of these proteins could be functionally expressed, and so their function remained uncertain. This situation changed when the Jentsch lab created a ClC-7 knockout mouse(65). These mice are gravely ill, and die within 30 days of birth. Careful analysis of their pathology revealed severe osteopetrosis, or hypercalcification of bone, leading to growth retardation and deformation. This pathology results from a loss of function of bone-resorbing osteoclasts. In WT animals these cells form a large acidic compartment in contact with the bone matrix (the "ruffled border"). In the KO animals osteoclasts develop, but do not acidify the ruffled border (based on acridine orange fluorescence); therefore they do not resorb bone. The authors noted that the widely expressed CIC-7 is localized to lysosomes in many cell types, and since the ruffled border is formed from fusion of acidic "lysosome related organelles" to the plasma membrane, they suggested that ClC-7 contributes the counterion pathway in ruffled border and, by analogy, in lysosomes as well. Both severe and benign human osteopetroses can also result from mutations in CIC-7(65, 67-71).

The hypothesis that $\mathrm{CIC}-7$ is part of the lysosomal counterion pathway was tested by Graves et al. using isolated HeLa cell lysosomes and intact HeLa cells(33). Since ClC-7 is ubiquitously expressed (as would be expected for a counterion conductance) the authors used siRNA to transiently knock down baseline $\mathrm{ClC}-7$ expression. HeLa cells lysosomes have a $\mathrm{Cl}^{-} / \mathrm{H}^{+}$antiporter activity similar to the one described above in rat liver lysosomes. Knockdown of $\mathrm{ClC}-7$ expression with siRNA abolished this antiport activity. If this antiporter participates in the counterion pathway, then its knockdown (or inhibition) should inhibit lysosomal acidification. Indeed, staining living HeLa cells with Lysotracker Green (a weak base similar to acridine orange that concentrates in and stains acidic organelles) showed reduced staining in CIC-7 knockout cells compared with either WT or control siRNA transfected cells(33). This reduced staining suggests a reduction in lysosomal acidity in the KO cells, as predicted if $\mathrm{ClC}-7$ is a major part of the counterion pathway in these cells.

Unfortunately, further results from CIC-7 KO mice complicate the picture. Several sets of quantitative measurements (using Oregon Green 488-dextran) on cells from these animals $(34,72,73)$ 
reveal no significant change in lysosomal pH for the CIC-7 KO compared with WT cells. Also, cells defective in Ostm 1, a $\beta$-subunit needed for proper targeting of $\mathrm{ClC}-7$ to lysosomes, also maintain the acidic $\mathrm{pH}$ in their lysosomes(73). What could account for these differences? The dye used in the HeLa lysosome experiments has been criticized for its qualitative nature(74), however, like acridine orange, such dyes can be useful for simple conclusions. Furthermore, similar experiments with ratiometric dextran dyes yield similar results (Lioi and Mindell, unpublished observations). There are several other explanations that must be considered for these differences. First, knockout animals are well-known to compensate for the defects due to their lost protein expression $(75,76)$. It is possible that the mice have $\mathrm{pH}$ regulatory mechanisms in their lysosomes that allow them to adapt other transport proteins to maintain $\mathrm{pH}$. Indeed, the Jentsch lab found increased levels of retargeting of $\mathrm{ClC}-3$ and $\mathrm{ClC}-6$ in lysosomal fractions from brain in the $\mathrm{ClC}-7 \mathrm{KO}(77)$. Also, it is important to note that these experiments have been performed in different cell types. Lysosomes in different tissues may well use different combinations of channels and transporters to maintain $\mathrm{pH}$ in the context of the very different digestive demands placed on them. Little is known about how acidification might be tuned in a tissue specific manner.

Given the lack of $\mathrm{pH}$ change in $\mathrm{ClC}-7 \mathrm{KO}$ cells, it is surprising that other functional changes are apparent. Wartosch et al. (78) injected a fluorescently labeled protein ( $\beta$-lactoglobulin) into mice with a kidney specific ClC-7 knockout. In these animals the kidneys are chimeric for the ClC-7 KO. They sacrificed the animals at varying times and monitored the degradation and release of the labeled protein both using western blots and imaging of tissue slices. They found that though the labeled protein was delivered efficiently to the lysosomes, the $\mathrm{CIC}-7 \mathrm{KO}$ cells in the kidney were substantially slower at degrading the $\beta$-lactoglobulin than were the WT cells. Given the lack of a pH change in their experiments, the authors suggested that the role of $\mathrm{ClC}-7$ could be to utilize the $\mathrm{pH}$ gradient to maintain $\mathrm{Cl}^{-}$in the lysosome at higher concentrations than its equilibrium concentration. They point out that Cathepsin C, a lysosomal protease, has been reported to show $\left[\mathrm{Cl}^{-}\right]$-dependent activity(79), raising the possibility that regulation of intralysosomal $\mathrm{Cl}^{-}$could be an important role for $\mathrm{ClC}-7$.

In summary, there is broad agreement that $\mathrm{ClC}-7$ is a $2 \mathrm{Cl}^{-} / 1 \mathrm{H}^{+}$antiporter localized in the lysosomal membrane and that it contributes to the observed $\mathrm{Cl}^{-}$permeability of the lysosomal membrane. How much it contributes to the essential counterion pathway or to regulating lysosomal $\left[\mathrm{Cl}^{-}\right]$remain open questions, however.

\section{Role of proton-chloride coupling in CIC-7 function}

Whatever the physiological task of $\mathrm{ClC}-7$, one of the remarkable observations regarding the intracellular $\mathrm{ClCs}$ concerns the role of coupled $\mathrm{H}^{+}-\mathrm{Cl}^{-}$transport in their function. We know that the $\mathrm{CLC}$ family includes both ion channels and antiporters (divided about half and half in mammals). Consistently, all of the channels function in the plasma membrane whereas all of the antiporters function in intracellular organelle membranes. Thus, one must conclude that there have been evolutionary pressures to maintain this sorting integrity. How is proton coupling related to $\mathrm{ClC}-7$ function? This question was recently addressed by the Jentsch lab, which created mice in which the WT CIC-7 antiporter was replaced with a mutant form of the protein lacking the essential "gating glutamate" (referred to as UNC, for uncoupled). This glutamate residue is essential for coupled $\mathrm{H}^{+} / \mathrm{Cl}^{-}$ transport $(58,61)$ in the CLC antiporters and for voltage-dependent gating $(80)$ in the CLC channels. Mutations at this site eliminate proton transport and essentially yield a passive $\mathrm{Cl}$ - uniporter(58)-a transporter that moves $\mathrm{Cl}$ only down its electrochemical gradient. Remarkably, mice carrying this mutant transporter recapitulate much of the phenotype of the total CIC-7 knockout(81), including osteopetrosis, growth retardation, and accumulation of lysosomal storage material, though the phenotype is milder than the full KO. Analysis of acidification in lysosomes from the mutant mice revealed a complex picture. The organelles could not support measurable $\mathrm{Cl}^{-} / \mathrm{H}^{+}$antiport, but could 
still acidify, as measured using Oregon Green dextran fluorescence. Indeed in an in vitro assay on isolated lysosomes, the uncoupled mutants seems to acidify to a slightly lower $\mathrm{pH}$ in the uncoupled lysosomes compared with WT, whereas the full KO acidified to a slightly higher $\mathrm{pH}$. In living cells, however, lysosomal pH appeared identical in cells from WT, KO and UNC mice. Experiments using a novel fluorescent $\mathrm{Cl}$ indicator dye hint at higher luminal $\left[\mathrm{Cl}^{-}\right]$in the $\mathrm{UNC}$ and $\mathrm{KO}$ lysosomes, but since the dye is not calibrated to known $\mathrm{Cl}^{-}$concentrations it is difficult to interpret these results.

This work presents a complex and difficult picture to interpret. It is clear that the UNC ClC-7 causes almost as severe a phenotype as the complete KO. Taken as a whole, the data support both models of $\mathrm{ClC}-7$ function, as either a counterion pathway, a $\mathrm{Cl}^{-}$concentrating mechanism, or both.

\section{New possibilities: active regulation of lysosomal pH}

Recent work from the Maxfield lab may help clarify the role of $\mathrm{CIC}-7$ as well as expanding our understanding of the dynamic features of lysosomal acidification. Majumdar and coworkers noted that conflicting results had been reported regarding the degradation of amyloid $A \beta$, a key molecule in the pathology of Alzheimer's disease: where primary cultures of microglia (CNS macrophages) could internalize $A \beta$ peptide, but could not degrade it, either microglia activated by passive immunization or macrophages can both internalize and degrade the $A \beta$ peptide( 82 ). Investigating this difference Majumdar et al. found that microglia actually contain higher levels of lysosomal proteases than similar macrophages but that the microglia lysosomes were substantially more basic than those of macrophages ( 6 vs. 5)(83). However, when the microglia were activated by treatment with macrophage colony-stimulating factor (MCSF) or Interleukin-6 (II-6) their lysosomal pH dropped to $\sim 5$ and they became more effective at digesting $A \beta$ peptide (83). Probing the mechanism of this effect, the authors further found that $\mathrm{CIC}-7$ in the quiescent microglia is primarily not targeted to lysosomes; instead it was apparently destined for degradation by the proteasome(84). However, upon activation $\mathrm{CIC}-7$ was recruited to lysosomes. Further, knockdown of $\mathrm{ClC}-7$ expression with siRNA prevented the MCSF induced retargeting of $\mathrm{ClC}-7$, reducduction of $A \beta$ degradation, and lowering of $\mathrm{pH}(84)$.

These results have profound implications for our appreciation of the subtleties of lysosomal acidification. First, they provide further evidence that $\mathrm{CIC}-7$ is an important part of the acidification mechanism, presumeably due to its role as a counterion pathway. In addition, they suggest that lysosomal acidification is more dynamic and more regulated that previously considered.

\section{Candidates for the cation pathway}

Where there are well-established candidate transporters for the anion moving portion of the lysosomal counterion pathway, candidates for the cation transporting component are more tentative. One candidate is the cation channel TRPML1. This channel is a member of the TRP (for Transient Receptor Potential, reflecting the effect on drosophila retina of mutating the founding member) channel family, which also includes the channels that sense heat and cold, among many others(85). The TRP channels are tetrameric cation channels with a 6-transmembrane domain architecture similar to the voltage gated $\mathrm{K}^{+}, \mathrm{Na}^{+}$, and $\mathrm{Ca}^{2+}$ channels and are activated by a wide range of stimuli including G-protein-coupled receptor interactions, ligand activation, and temperature(85). TRPML1 is encoded by the MCOLN1 gene, which is mutated in the lysosomal storage disorder, Mucolipidosis type IV (MLIV). This autosomal recessive disease is characterized by a slowly progressing neurodegenerative phenotype(86). The TRPML1 protein is localized to lysosomes and its disruption (in MLIV patient fibroblasts) has been reported to raise (87), maintain (88), or even lower(89) lysosomal pH, creating confusion as to its role in the acidification process. Furthermore, TRPML1 has also been reported to mediate iron relase from endolysosomes(90) and to play a role in lysosomal $\mathrm{Ca}^{2+}$ release(91) and its dysfunction leads to a range of other lysosomal phenotypes. Clearly, the actual role of TRPML1 remains to be clearly defined, though it may well serve multiple of its proposed roles. Finally, a "two- 
pore" channel, TPC2, has been reported to localize to lysosomes and to play a role in $\mathrm{Ca}^{2+}$ release from the organelles(92). Whether this channel has a role in $\mathrm{pH}$ regulation remains to be seen.

\section{Conclusions}

In conclusion, though many of the molecular players in lysosomal acidificaion (and organellar acidificaiton in general) are now clear, the mechanisms by which these transporters work together (along with lumenal buffers, membrane voltage and other factors) remain unclear. Furthermore, new possibilities, like the dynamic regulation of lysosomal $\mathrm{pH}$, are emerging and must be incorporated into any comprehensive framework. 


\section{Points made:}

1. Multiple factors influence lysosomal acidificaion

2. V-ATPases are the primary drivers of acidificationj, converting metabolic energy into proton gradients. Maximally efficient ATPases could acidify organelles to lower $\mathrm{pHs}$ than are observed in most compartments.

3. Because the V-ATPase is electrogenic, counterions must move to dissipate voltage and facilitate bulk proton transport.

4. Extensive functional analysis on isolated lysosomes clearly demonstrates both anion and cation permeabilities, though the anion pathway is more consistently observed.

5. CFTR is unlikely to provide the anion pathway in most lysosomes

6. CIC-7 is a good candidate for the anion pathway, but open questions remain as to its relevance, particularly the lack of $\mathrm{pH}$ change in knockout mouse lysosomes.

7. No cation channel has emerged as a consensus candidate for a cation-selective counterion pathway.

\section{Future directions:}

1. Clarify roles of V-ATPase isoforms

2. Quantitatively determine the relative contributions of anion and cation transport to the counterion pathway

3. Explore tissue specific differences in acidification mechanisms

4. Determine the molecular basis of the lysosomal cation permeability

5. How does the proton-dependent transport of substrates out of lysosomes affect the acidification process? 


\section{Bibliography}

1. Metchnikoff E. 1893. Lectures on the comparative pathology of inflammation (reprinted 1968). New York: Dover

2. Coffey JW, De Duve C. 1968. Digestive activity of lysosomes. I. The digestion of proteins by extracts of rat liver lysosomes, J Biol Chem 243: 3255-63

3. Ohkuma S, Moriyama Y, Takano T. 1982. Identification and characterization of a proton pump on lysosomes by fluorescein-isothiocyanate-dextran fluorescence, Proc Natl Acad Sci U S A 79: 2758-62

4. Forgac M. 2007. Vacuolar ATPases: rotary proton pumps in physiology and pathophysiology, Nat Rev Mol Cell Biol 8: 917-29

5. Hirata T, Iwamoto-Kihara A, Sun-Wada GH, Okajima T, Wada Y, Futai M. 2003. Subunit rotation of vacuolar-type proton pumping ATPase: relative rotation of the $G$ and $C$ subunits, J Biol Chem 278: $23714-9$

6. Yokoyama K, Nakano M, Imamura H, Yoshida M, Tamakoshi M. 2003. Rotation of the proteolipid ring in the V-ATPase, J Biol Chem 278: 24255-8

7. Arai H, Terres G, Pink S, Forgac M. 1988. Topography and subunit stoichiometry of the coated vesicle proton pump, J Biol Chem 263: 8796-802

8. Johnson RG, Beers MF, Scarpa A. 1982. H+ ATPase of chromaffin granules. Kinetics, regulation, and stoichiometry, J Biol Chem 257: 10701-7

9. Schmidt AL, Briskin DP. 1993. Reversal of the red beet tonoplast H(+)-ATPase by a pyrophosphate-generated proton electrochemical gradient, Arch Biochem Biophys 306: 407-14

10. Grabe M, Wang H, Oster G. 2000. The mechanochemistry of V-ATPase proton pumps, Biophys J 78: 2798-813

11. Muller ML, Jensen M, Taiz L. 1999. The vacuolar H+-ATPase of lemon fruits is regulated by variable H+/ATP coupling and slip, J Biol Chem 274: 10706-16

12. Ohkuma S, Moriyama Y, Takano T. 1983. Electrogenic nature of lysosomal proton pump as revealed with a cyanine dye, $J$ Biochem 94: 1935-43

13. Harikumar P, Reeves JP. 1983. The lysosomal proton pump is electrogenic, J Biol Chem 258: 10403-10

14. Hedrich R, Kurkdjian A, Guern J, Flugge UI. 1989. Comparative studies on the electrical properties of the $\mathrm{H}+$ translocating ATPase and pyrophosphatase of the vacuolar-lysosomal compartment, EMBO J 8: 2835-41

15. Kettner C, Bertl A, Obermeyer G, Slayman C, Bihler H. 2003. Electrophysiological analysis of the yeast $V$-type proton pump: variable coupling ratio and proton shunt, Biophys $J$ 85: 3730-8

16. Wieczorek H, Grber G, Harvey WR, Huss M, Merzendorfer H, Zeiske W. 2000. Structure and regulation of insect plasma membrane $\mathrm{H}(+)$ V-ATPase, J Exp Biol 203: 127-35

17. Kane PM. 1995. Disassembly and Reassembly of the Yeast Vacuolar H+-ATPase in Vivo, Journal of Biological Chemistry 270: 17025-32

18. Seol JH, Shevchenko A, Deshaies RJ. 2001. Skp1 forms multiple protein complexes, including RAVE, a regulator of V-ATPase assembly, Nat Cell Biol 3: 384-91

19. Smardon AM, Tarsio M, Kane PM. 2002. The RAVE complex is essential for stable assembly of the yeast V-ATPase, J Biol Chem 277: 13831-9

20. Smardon AM, Kane PM. 2007. RAVE is essential for the efficient assembly of the $C$ subunit with the vacuolar $\mathrm{H}(+)$-ATPase, J Biol Chem 282: 26185-94

21. Lu M, Ammar D, Ives H, Albrecht F, Gluck SL. 2007. Physical interaction between aldolase and vacuolar $\mathrm{H}+-$ ATPase is essential for the assembly and activity of the proton pump, $\mathrm{J} \mathrm{Biol}$ Chem 282: $24495-503$ 
22. Xu T, Forgac M. 2001. Microtubules are involved in glucose-dependent dissociation of the yeast vacuolar [H+]-ATPase in vivo, J Biol Chem 276: 24855-61

23. Manolson MF, Proteau D, Preston RA, Stenbit A, Roberts BT, et al. 1992. The VPH1 gene encodes a 95-kDa integral membrane polypeptide required for in vivo assembly and activity of the yeast vacuolar $\mathrm{H}(+)$-ATPase, J Biol Chem 267: 14294-303

24. Manolson MF, Wu B, Proteau D, Taillon BE, Roberts BT, et al. 1994. STV1 gene encodes functional homologue of 95-kDa yeast vacuolar $\mathrm{H}(+)$-ATPase subunit Vph1 p, J Biol Chem 269: 14064-74

25. Kawasaki-Nishi S, Nishi T, Forgac M. 2001. Yeast V-ATPase complexes containing different isoforms of the $100-\mathrm{kDa}$ a-subunit differ in coupling efficiency and in vivo dissociation, $J$ Biol Chem 276: 17941-8

26. Toei M, Saum R, Forgac M. 2010. Regulation and isoform function of the V-ATPases, Biochemistry 49: 4715-23

27. Smith AN, Jouret F, Bord S, Borthwick KJ, Al-Lamki RS, et al. 2005. Vacuolar H+-ATPase d2 subunit: molecular characterization, developmental regulation, and localization to specialized proton pumps in kidney and bone, J Am Soc Nephrol 16: 1245-56

28. Lee SH, Rho J, Jeong D, Sul JY, Kim T, et al. 2006. v-ATPase V0 subunit d2-deficient mice exhibit impaired osteoclast fusion and increased bone formation, Nat Med 12: 1403-9

29. Karet FE, Finberg KE, Nelson RD, Nayir A, Mocan H, et al. 1999. Mutations in the gene encoding B1 subunit of H+-ATPase cause renal tubular acidosis with sensorineural deafness, Nat Genet 21: $84-90$

30. Dell'Antone P. 1979. Evidence for an ATP-driven "proton pump" in rat liver lysosomes by basic dyes uptake, Biochem Biophys Res Commun 86: 180-9

31. Cuppoletti J, Aures-Fischer D, Sachs G. 1987. The lysosomal H+ pump: 8-azido-ATP inhibition and the role of chloride in $\mathrm{H}+$ transport, Biochim Biophys Acta 899: 276-84

32. Van Dyke RW. 1993. Acidification of rat liver lysosomes: quantitation and comparison with endosomes, Am J Physiol 265: C901-17

33. Graves AR, Curran PK, Smith $\mathrm{CL}$, Mindell JA. 2008. The $\mathrm{Cl}(-) / \mathrm{H}(+)$ antiporter $\mathrm{ClC}-7$ is the primary chloride permeation pathway in lysosomes, Nature 453: 788-92

34. Steinberg BE, Huynh KK, Brodovitch A, Jabs S, Stauber T, et al. 2010. A cation counterflux supports lysosomal acidification, J Cell Biol 189: 1171-86

35. Pelegrin P, Barroso-Gutierrez C, Surprenant A. 2008. P2X7 receptor differentially couples to distinct release pathways for IL-1 beta in mouse macrophage, $J$ Immunol 180: 7147-57

36. Pelegrin P, Surprenant A. 2006. Pannexin-1 mediates large pore formation and interleukin1beta release by the ATP-gated $\mathrm{P} 2 \mathrm{X} 7$ receptor, EMBO J 25: 5071-82

37. Berg TO, Stromhaug E, Lovdal T, Seglen O, Berg T. 1994. Use of glycyl-L-phenylalanine 2naphthylamide, a lysosome-disrupting cathepsin $C$ substrate, to distinguish between lysosomes and prelysosomal endocytic vacuoles, Biochem J 300 ( Pt 1): 229-36

38. McGuinness L, Bardo SJ, Emptage NJ. 2007. The lysosome or lysosome-related organelle may serve as a Ca2+ store in the boutons of hippocampal pyramidal cells, Neuropharmacology 52: 126-35

39. Jones PM, O'Mara ML, George AM. 2009. ABC transporters: a riddle wrapped in a mystery inside an enigma, Trends Biochem Sci 34: 520-31

40. Gadsby DC, Vergani P, Csanady L. 2006. The ABC protein turned chloride channel whose failure causes cystic fibrosis, Nature 440: 477-83

41. Haggie PM, Verkman AS. 2009. Defective organellar acidification as a cause of cystic fibrosis lung disease: reexamination of a recurring hypothesis, Am J Physiol Lung Cell Mol Physiol 296: L859-67 
42. Barasch J, Kiss B, Prince A, Saiman L, Gruenert D, al-Awqati Q. 1991. Defective acidification of intracellular organelles in cystic fibrosis, Nature 352: 70-3

43. Di A, Brown ME, Deriy LV, Li C, Szeto FL, et al. 2006. CFTR regulates phagosome acidification in macrophages and alters bactericidal activity, Nat Cell Biol 8: 933-44

44. Deriy LV, Gomez EA, Zhang G, Beacham DW, Hopson JA, et al. 2009. Disease-causing mutations in the cystic fibrosis transmembrane conductance regulator determine the functional responses of alveolar macrophages, J Biol Chem 284: 35926-38

45. Seksek O, Biwersi J, Verkman AS. 1996. Evidence against defective trans-Golgi acidification in cystic fibrosis, J Biol Chem 271: 15542-8

46. Biwersi J, Verkman AS. 1994. Functional CFTR in endosomal compartment of CFTR-expressing fibroblasts and T84 cells, Am J Physiol 266: C149-56

47. Lukacs GL, Chang XB, Kartner N, Rotstein OD, Riordan JR, Grinstein S. 1992. The cystic fibrosis transmembrane regulator is present and functional in endosomes. Role as a determinant of endosomal pH, J Biol Chem 267: 14568-72

48. Haggie PM, Verkman AS. 2007. Cystic fibrosis transmembrane conductance regulatorindependent phagosomal acidification in macrophages, J Biol Chem 282: 31422-8

49. Haggie PM, Verkman AS. 2009. Unimpaired lysosomal acidification in respiratory epithelial cells in cystic fibrosis, J Biol Chem 284: 7681-6

50. White MM, Miller C. 1979. A voltage-gated anion channel from the electric organ of Torpedo californica, J Biol Chem 254: 10161-6

51. Jentsch TJ, Steinmeyer K, Schwarz G. 1990. Primary structure of Torpedo marmorata chloride channel isolated by expression cloning in Xenopus oocytes, Nature 348: 510-4

52. Jentsch TJ. 2008. CLC chloride channels and transporters: from genes to protein structure, pathology and physiology, Crit Rev Biochem Mol Biol 43: 3-36

53. Planells-Cases R, Jentsch TJ. 2009. Chloride channelopathies, Biochim Biophys Acta 1792: $173-$ 89

54. Dutzler R, Campbell EB, Cadene M, Chait BT, MacKinnon R. 2002. X-ray structure of a ClC chloride channel at 3.0 A reveals the molecular basis of anion selectivity, Nature 415: 287-94

55. Feng L, Campbell, E. B., Hsiung, Y. Mackinnon R. . 2010. Structure of a eukaryotic CLC transporter defines an intermediate state in the transport cycle, Science

56. Miller C. 1982. Open-state substructure of single chloride channels from Torpedo electroplax, Philos Trans R Soc Lond B Biol Sci 299: 401-11

57. Middleton RE, Pheasant DJ, Miller C. 1996. Homodimeric architecture of a CIC-type chloride ion channel, Nature 383: 337-40

58. Accardi A, Miller C. 2004. Secondary active transport mediated by a prokaryotic homologue of ClC Cl- channels, Nature 427: 803-7

59. Accardi A, Kolmakova-Partensky L, Williams C, Miller C. 2004. Ionic currents mediated by a prokaryotic homologue of CLC Cl- channels, J Gen Physiol 123: 109-19

60. Scheel O, Zdebik AA, Lourdel S, Jentsch TJ. 2005. Voltage-dependent electrogenic chloride/proton exchange by endosomal CLC proteins, Nature 436: 424-7

61. Picollo A, Pusch M. 2005. Chloride/proton antiporter activity of mammalian CLC proteins CIC-4 and CIC-5, Nature 436: 420-3

62. Gunther W, Luchow A, Cluzeaud F, Vandewalle A, Jentsch TJ. 1998. CIC-5, the chloride channel mutated in Dent's disease, colocalizes with the proton pump in endocytotically active kidney cells, Proc Natl Acad Sci U S A 95: 8075-80

63. Mohammad-Panah R, Harrison R, Dhani S, Ackerley C, Huan LJ, et al. 2003. The chloride channel CIC-4 contributes to endosomal acidification and trafficking, J Biol Chem 278: 2926777 
64. Piwon N, Gunther W, Schwake M, BosI MR, Jentsch TJ. 2000. CIC-5 Cl-channel disruption impairs endocytosis in a mouse model for Dent's disease, Nature 408: 369-73

65. Kornak U, Kasper D, Bosl MR, Kaiser E, Schweizer M, et al. 2001. Loss of the CIC-7 chloride channel leads to osteopetrosis in mice and man, Cell 104: 205-15

66. Brandt S, Jentsch TJ. 1995. CIC-6 and CIC-7 are two novel broadly expressed members of the CLC chloride channel family, FEBS Lett 377: 15-20

67. Cleiren E, Benichou O, Van Hul E, Gram J, Bollerslev J, et al. 2001. Albers-Schonberg disease (autosomal dominant osteopetrosis, type II) results from mutations in the CICN7 chloride channel gene, Hum Mol Genet 10: 2861-7

68. Campos-Xavier AB, Saraiva JM, Ribeiro LM, Munnich A, Cormier-Daire V. 2003. Chloride channel 7 (CLCN7) gene mutations in intermediate autosomal recessive osteopetrosis, Hum Genet 112: 186-9

69. Henriksen K, Gram J, Schaller S, Dahl BH, Dziegiel MH, et al. 2004. Characterization of osteoclasts from patients harboring a G215R mutation in CIC-7 causing autosomal dominant osteopetrosis type II, Am J Pathol 164: 1537-45

70. Frattini A, Pangrazio A, Susani L, Sobacchi C, Mirolo M, et al. 2003. Chloride channel CICN7 mutations are responsible for severe recessive, dominant, and intermediate osteopetrosis, $J$ Bone Miner Res 18: 1740-7

71. Kornak U, Ostertag A, Branger S, Benichou O, de Vernejoul MC. 2006. Polymorphisms in the CLCN7 gene modulate bone density in postmenopausal women and in patients with autosomal dominant osteopetrosis type II, J Clin Endocrinol Metab 91: 995-1000

72. Kasper D, Planells-Cases R, Fuhrmann JC, Scheel O, Zeitz O, et al. 2005. Loss of the chloride channel CIC-7 leads to lysosomal storage disease and neurodegeneration, Embo J 24: 1079-91

73. Lange PF, Wartosch L, Jentsch TJ, Fuhrmann JC. 2006. CIC-7 requires Ostm1 as a beta-subunit to support bone resorption and lysosomal function, Nature 440: 220-3

74. DiCiccio JE, Steinberg BE. 2011. Lysosomal pH and analysis of the counter ion pathways that support acidification, J Gen Physiol 137: 385-90

75. Satyanarayana A, Kaldis P. 2009. Mammalian cell-cycle regulation: several Cdks, numerous cyclins and diverse compensatory mechanisms, Oncogene 28: 2925-39

76. Rudmann DG, Durham SK. 1999. Utilization of Genetically Altered Animals in the Pharmaceutical Industry, Toxicologic Pathology 27: 111-4

77. Poet M, Kornak U, Schweizer M, Zdebik AA, Scheel O, et al. 2006. Lysosomal storage disease upon disruption of the neuronal chloride transport protein CIC-6, Proc Natl Acad Sci U SA 103: 13854-9

78. Wartosch L, Fuhrmann JC, Schweizer M, Stauber T, Jentsch TJ. 2009. Lysosomal degradation of endocytosed proteins depends on the chloride transport protein CIC-7, FASEB J 23: 4056-68

79. Cigic B, Pain RH. 1999. Location of the binding site for chloride ion activation of cathepsin C, Eur J Biochem 264: 944-51

80. Dutzler R, Campbell EB, MacKinnon R. 2003. Gating the selectivity filter in CIC chloride channels, Science 300: 108-12

81. Weinert S, Jabs S, Supanchart C, Schweizer M, Gimber N, et al. 2010. Lysosomal pathology and osteopetrosis upon loss of $\mathrm{H}+-$ driven lysosomal $\mathrm{Cl}$ - accumulation, Science 328: 1401-3

82. Majumdar A, Chung H, Dolios G, Wang R, Asamoah N, et al. 2008. Degradation of fibrillar forms of Alzheimer's amyloid beta-peptide by macrophages, Neurobiol Aging 29: 707-15

83. Majumdar A, Cruz D, Asamoah N, Buxbaum A, Sohar I, et al. 2007. Activation of microglia acidifies lysosomes and leads to degradation of Alzheimer amyloid fibrils, Mol Biol Cell 18: 1490-6 
84. Majumdar A, Capetillo-Zarate E, Cruz D, Gouras GK, Maxfield FR. 2011. Degradation of Alzheimer's amyloid fibrils by microglia requires delivery of $\mathrm{ClC}-7$ to lysosomes, $\mathrm{Mol}$ Biol Cell 22: 1664-76

85. Ramsey IS, Delling M, Clapham DE. 2006. An introduction to TRP channels, Annu Rev Physiol 68: 619-47

86. Altarescu G, Sun M, Moore DF, Smith JA, Wiggs EA, et al. 2002. The neurogenetics of mucolipidosis type IV, Neurology 59: 306-13

87. Bach G, Chen CS, Pagano RE. 1999. Elevated lysosomal pH in Mucolipidosis type IV cells, Clin Chim Acta 280: 173-9

88. Pryor PR, Reimann F, Gribble FM, Luzio JP. 2006. Mucolipin-1 is a lysosomal membrane protein required for intracellular lactosylceramide traffic, Traffic 7: 1388-98

89. Soyombo AA, Tjon-Kon-Sang S, Rbaibi Y, Bashllari E, Bisceglia J, et al. 2006. TRP-ML1 Regulates Lysosomal $\mathrm{pH}$ and Acidic Lysosomal Lipid Hydrolytic Activity, Journal of Biological Chemistry 281: 7294-301

90. Dong XP, Cheng X, Mills E, Delling M, Wang F, et al. 2008. The type IV mucolipidosis-associated protein TRPML1 is an endolysosomal iron release channel, Nature 455: 992-6

91. Zhang F, Jin S, Yi F, Li P-L. 2009. TRP-ML1 functions as a lysosomal NAADP-sensitive Ca2+ release channel in coronary arterial myocytes, Journal of Cellular and Molecular Medicine 13: 3174-85

92. Calcraft PJ, Ruas M, Pan Z, Cheng X, Arredouani A, et al. 2009. NAADP mobilizes calcium from acidic organelles through two-pore channels, Nature 459: 596-600 


\section{Figure Legends}

Figure 1. Interior pHs of intracellular organelles. $\mathrm{pH}$ gradually drops along the endocytic pathway (black organelles) from early endosomes, to late endosomes, to lysosomes, which are most acidic. The opposite occurs along the secretory pathway, with the Golgi apparatus beween $\mathrm{pH} 6.9$ and 6.0 (cis to trans) and secretory vesicles more acidic ( $\mathrm{pH} 5.5$ ).

Figure 2. Lysosomal transporters involved in $\mathrm{pH}$ homeostasis. The V-type ATPase (green) uses the metabolic energy of ATP hydrolysis to drive protons into the lumen. This process builds a net positive charge inside the lumen of the lysosome which can be dissipated by $\mathrm{K}^{+}$efflux through a cation channel or transporter (blue) or by $\mathrm{Cl}^{-}$influx through $\mathrm{ClC}-7, \mathrm{a} \mathrm{Cl} / \mathrm{H}^{+}$antiporter.

Figure 3. Structure of the V-type proton ATPase. The soluble $V_{1}$ complex (red) is made up of ATP hydrolyzing A and B subunits. The rotor, made up of $c$, $c^{\prime}$ and $c^{\prime \prime}$ (green) is embedded in the membrane and carries protons from uptake sites on the cytoplasmic side to release sites on the lumenal side of the membrane. The a subunit (brown) contains access pathways to both sides of the membrane as well as a critical, conserved arginine residue required for transport. The $D$ subunit of the $\mathrm{V}_{1}$ domain and $\mathrm{d}$ subunit of the $\mathrm{V}_{0}$ domain comprise the 'central stalk' connecting rotor to ATPase domains. $\mathrm{E}$ and $\mathrm{G}$ subunits make the peripheral stalks.

Figure 4. Phylogenetic tree of the mammalian CLC proteins. The antiporters are shown in blue and the channels in red, separated by a dashed line. For reference, $\mathrm{ClC}-\mathrm{ec} 1$, an antiporter from E. coli and $\mathrm{ClC}-0$, the first CLC discovered (from Torpedo) are also indicated. Physiological functions are noted where known. 


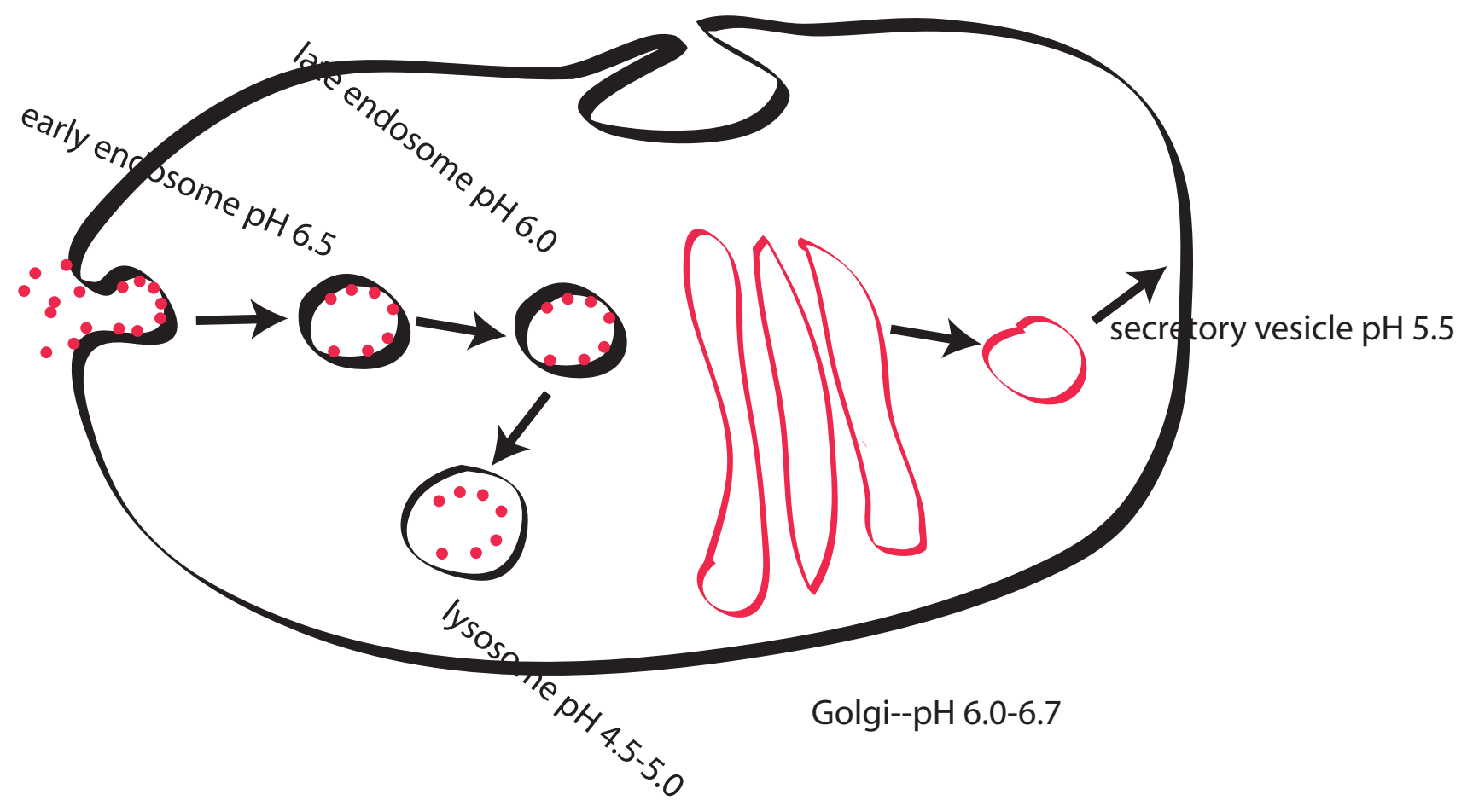

Figure 1 Interior pHs of intracellular organelles 


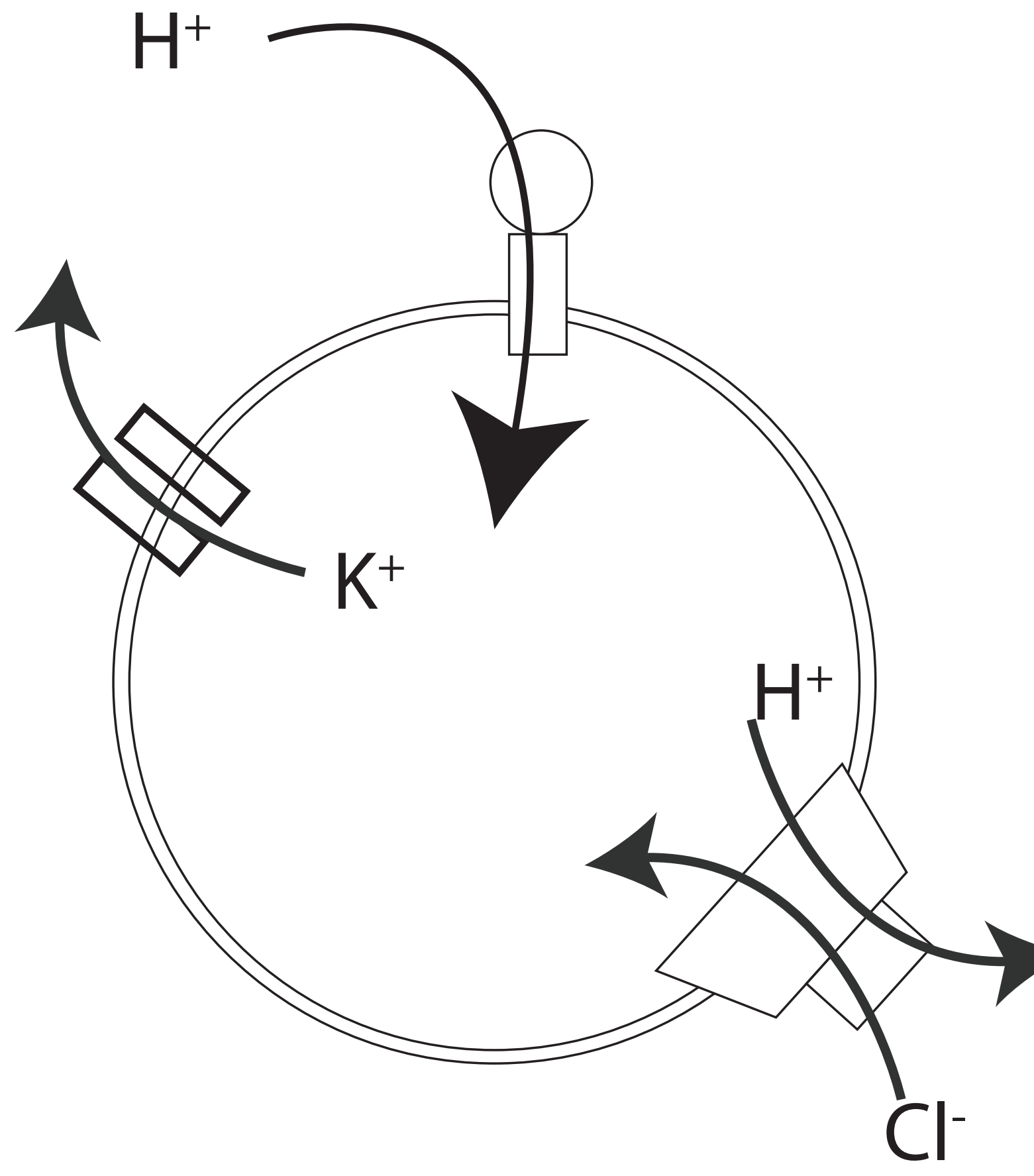




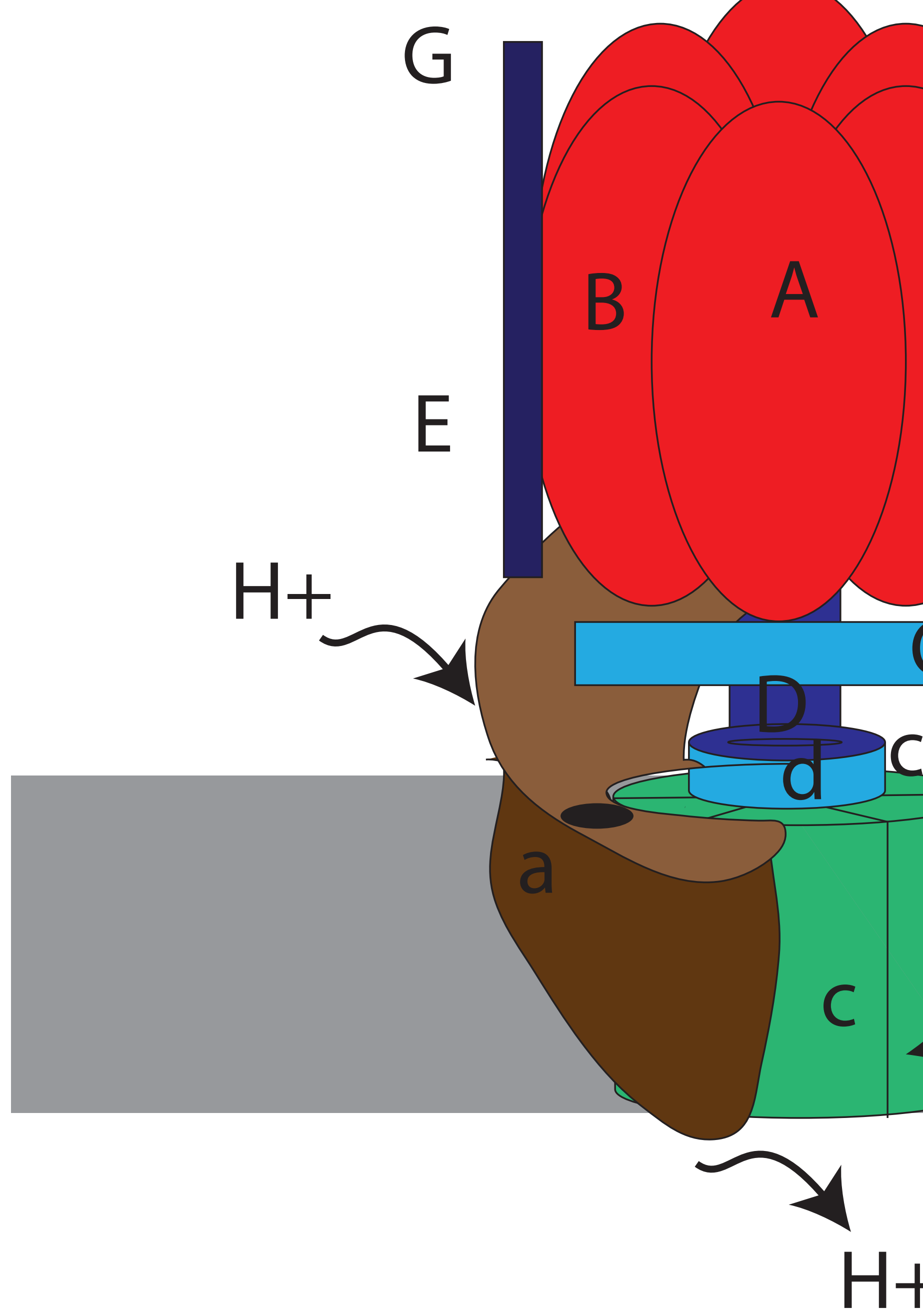




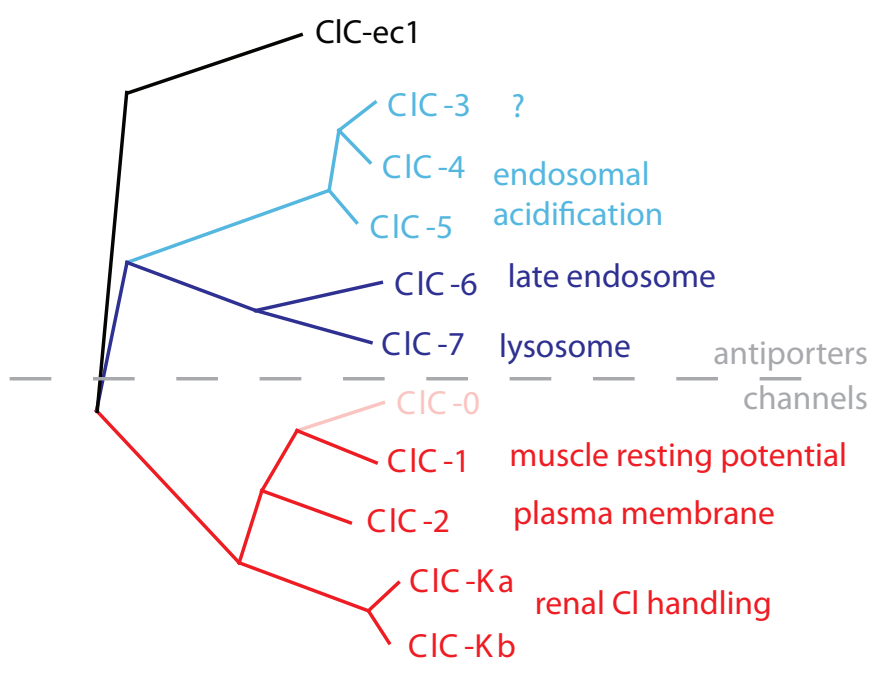

